\title{
Synthesis of wurtzite ZnSe nanorings by thermal evaporation
}

\author{
Y. P. Leung and Wallace C. H. Choy ${ }^{\mathrm{a})}$ \\ Department of Electrical and Electronic Engineering, University of Hong Kong, \\ Pokfulam Road, Hong Kong, China \\ I. Markov \\ Institute of Physical Chemistry, Bulgarian Academy of Sciences, 1113 Sofia, Bulgaria \\ G. K. H. Pang \\ Department of Applied Physics, Hong Kong Polytechnic University, Kowloon, \\ Hong Kong, China \\ H. C. Ong \\ Department of Physics, The Chinese University of Hong Kong, Shatin, Hong Kong, China \\ T. I. Yuk \\ Department of Electrical and Electronic Engineering, University of Hong Kong, \\ Pokfulam Road, Hong Kong, China
}

(Received 17 December 2005; accepted 15 March 2006; published online 4 May 2006)

\begin{abstract}
In this study, free standing crystalline ZnSe nanorings and nanowires have been fabricated on $\mathrm{Au}$ coated Si substrates by simple thermal evaporation of ZnSe powders. Ring- or wirelike morphology can be achieved in a controllable manner by using different reactor pressures during growth, while all the other conditions remain the same. Our results show that the ZnSe nanorings are wurtzite phase instead of the zinc-blende phase, observed in typical one-dimensional ZnSe nanostructures. The growth mechanism of the nanorings has been discussed, and the cathodoluminescence of the nanorings has been described. (C) 2006 American Institute of Physics. [DOI: 10.1063/1.2200155]
\end{abstract}

Semiconductors in quasi-one-dimensional (1D) forms have attracted a large amount of interests in the past decade due to their potential applications. They are considered to be ideal building blocks of functional nanoscale devices. ${ }^{1}$ Considerable efforts have been devoted to the synthesis of 1D elementary and binary semiconductors. The catalytic growth method is one of the popular methods to synthesize 1D nanostructures based on the well-known vapor-liquid-solid (VLS) mechanism. ${ }^{2}$ Growth techniques such as thermal evaporation, ${ }^{3}$ metal organic chemical vapor deposition (MOCVD), ${ }^{4}$ solvothermal process, ${ }^{5}$ and molecular beam epitaxy $^{6}(\mathrm{MBE})$ have been utilized to fabricate various types of $1 \mathrm{D}$ nanostructures such as wires, ${ }^{7}$ tubes, ${ }^{8}$ coaxial cables, ${ }^{9}$ and belts. ${ }^{10}$ Recently, a ringlike nanostructure has been reported by Kong and Wang. ${ }^{11}$

$\mathrm{ZnSe}$ is an important wide-band-gap II-VI semiconductor for applications in short-wavelength optoelectronic devices such as blue laser diodes, light-emitting diodes (LEDs), photodetectors, ${ }^{12-14}$ and window materials in the field of photovoltaics. ${ }^{15} \mathrm{ZnSe}$ can also be combined with other II-VI semiconductors such as $\mathrm{ZnO}$ taking the advantage of carrier transfer between two binary II-VI semiconductors with different energy levels for device applications. ${ }^{16,17}$ Recently, $\mathrm{ZnSe}$ nanowires, ${ }^{4,18}$ nanobelts, ${ }^{19}$ and $\mathrm{ZnSe}$ nanorods on in situ synthesized $\mathrm{ZnSe}$ grains ${ }^{20}$ have been reported. However, most of the reports on $\mathrm{ZnSe}$ nanostructures were focused on the zinc-blende structure, only a few on its wurtzite counterpart yet without detailed explanation. ${ }^{19,23}$ Here, we show the controlled growth of wurtzite ZnSe nanorings by simple thermal evaporation through changing the reactor pressure. The formation of the wurtzite phase and growth mechanism of the nanorings will be discussed.

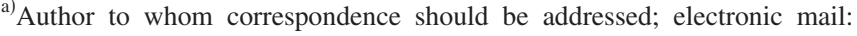
chchoy@eee.
}

The rings were grown in a tube furnace. About $0.5 \mathrm{~g}$ ZnSe powder (99.99\% Sigma Aldrich) was placed in an alumina boat, and the boat was then loaded to the center region of the furnace. A cleaned $\mathrm{Si}(100)$ substrate, with $\sim 5 \mathrm{~nm}$ thick $\mathrm{Au}$ sputtered on it, was placed about $30 \mathrm{~cm}$ downstream from the $\mathrm{ZnSe}$ source. The system was then evacuated for $30 \mathrm{~min}$ and purged with the carrier gas $(95 \% \mathrm{Ar}$ $+5 \% \mathrm{H}_{2}$ ) at a rate of about $400 \mathrm{SCCM}$ (SCCM denotes cubic centimeter per minute at STP) for $1 \mathrm{~h}$ to remove the residual oxygen. After that, the flow of the carrier gas was kept at 200 SCCM throughout the growth. The furnace were then heated up to $950{ }^{\circ} \mathrm{C}$ and maintained at this temperature for $30 \mathrm{~min}$. Various pressures were used to synthesize the nanostructures. After the growth, the system was cooled gradually to room temperature. A bright yellow colored product was obtained on the Si substrate after the growth.

The as-synthesis samples were characterized by transmission electron microscopy (TEM, JEOL 2010), field emission scanning electron microscopy (FE-SEM, LEO 1530) equipped with an energy dispersive x-ray spectroscopy (EDX), x-ray diffraction (XRD, Rigaku RU-300 with $\mathrm{Cu} K \alpha$ radiation), and cathodoluminescence (CL) system attached to SEM.

Figure 1(a) shows the SEM image of an as-synthesis sample grown at 0.2 torr in low magnification. It can be seen that ringlike structures have been grown. Figure 1(b) shows the higher magnification SEM image of a typical ring. It can be observed that the ring is formed by a close-loop belt. The rings usually have diameters of about few microns. The typical thickness of the rings is about $50-100 \mathrm{~nm}$. We find that the formation of the rings is sensitive to the growth pressure. When the growth is held at a pressure higher than or equal to 10 torr, zinc-blende structured nanowires (see Fig. 2) are grown instead of rings. The zinc-blende structure is confirmed by XRD as shown in the inset of Fig. 2. EDX analy- 


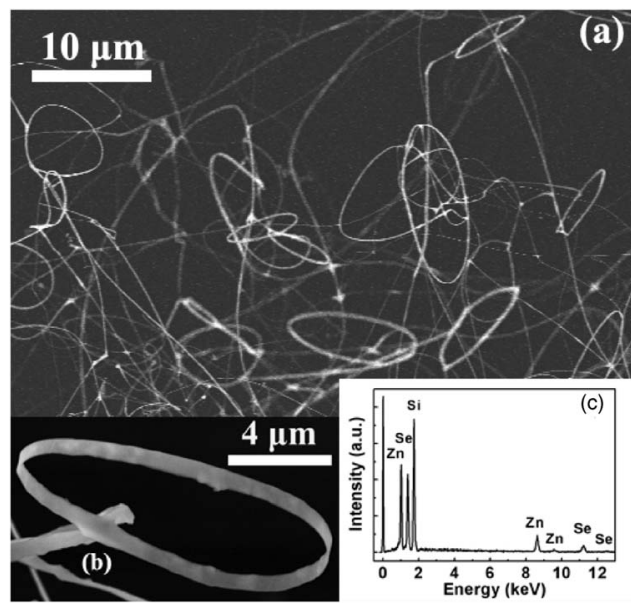

FIG. 1. (a) SEM image of the as-synthesis sample. Ringlike structures were grown. (b) Enlarged image of a typical single ring. (c) EDX spectrum of the nanorings.

ses of the rings have been conducted to study the composition of the rings and a typical spectrum is shown in Fig. 1(c). The results confirm that the rings are composed only of $\mathrm{Zn}$ and $\mathrm{Se}$ with a ratio about $1: 1$. The $\mathrm{Si}$ peak comes from the $\mathrm{Si}$ substrate. Meanwhile, selected area electron diffractions (SAEDs) have been investigated in TEM. Figure 3 shows a TEM image of a single ring. The insets are the SAED patterns of the corresponding regions of the ring as marked in the figure. The electron diffraction images indicate that, along the entire perimeter, the belt is bounded by (0001), $(11 \overline{2} 0)$, and $(\overline{1} 100)$ facets that the radial direction of the ring is always [0001], where the circular plane of the ring is [11̄̄0].

While ZnSe 1D nanostructures with zinc-blende structure have been widely reported, our results showed that $\mathrm{ZnSe}$ 1D nanorings with wurtzite structure have been synthesized. The wurtzite-zinc-blende (W-ZB) polytypism is common in compound semiconductors. Frequently, ZnSe exists in zincblende structure as stilleite in natural mines, ${ }^{21}$ while the wurtzite structure of $\mathrm{ZnSe}$ is a metastable phase. However, since there is only a small energy difference between the Wand $\mathrm{ZB}-\mathrm{ZnSe}(\sim 5.3 \mathrm{meV} / \mathrm{atom}),{ }^{22} \mathrm{~W}-\mathrm{ZnSe}$ can also be grown under suitable conditions. For example, Li et al. reported that a hexagon phase were found in $\mathrm{ZnSe}$ nanowires

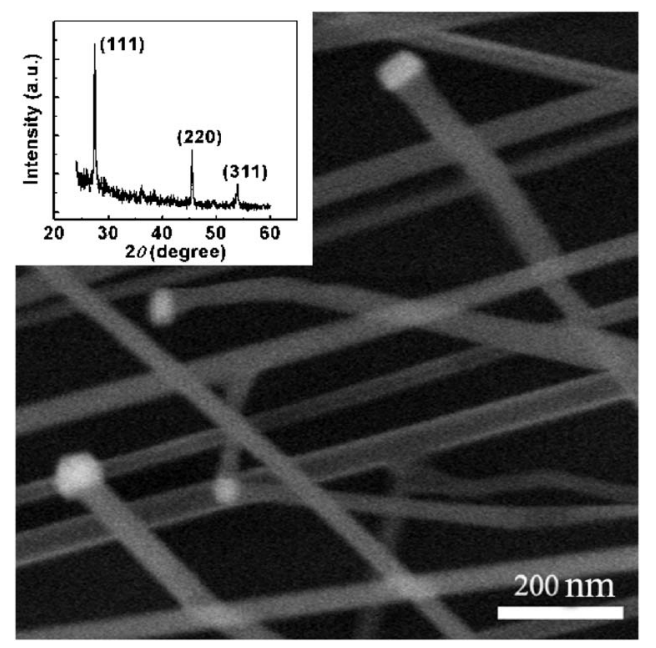

FIG. 2. Zinc-blende structured nanowires grown at 100 torr. The inset is the

XRD pattern of the nanowire, only ZnSe zinc-blende peaks present. Downloaded 31 Jul 2007 to 147.8.21.130. Redistribution subject

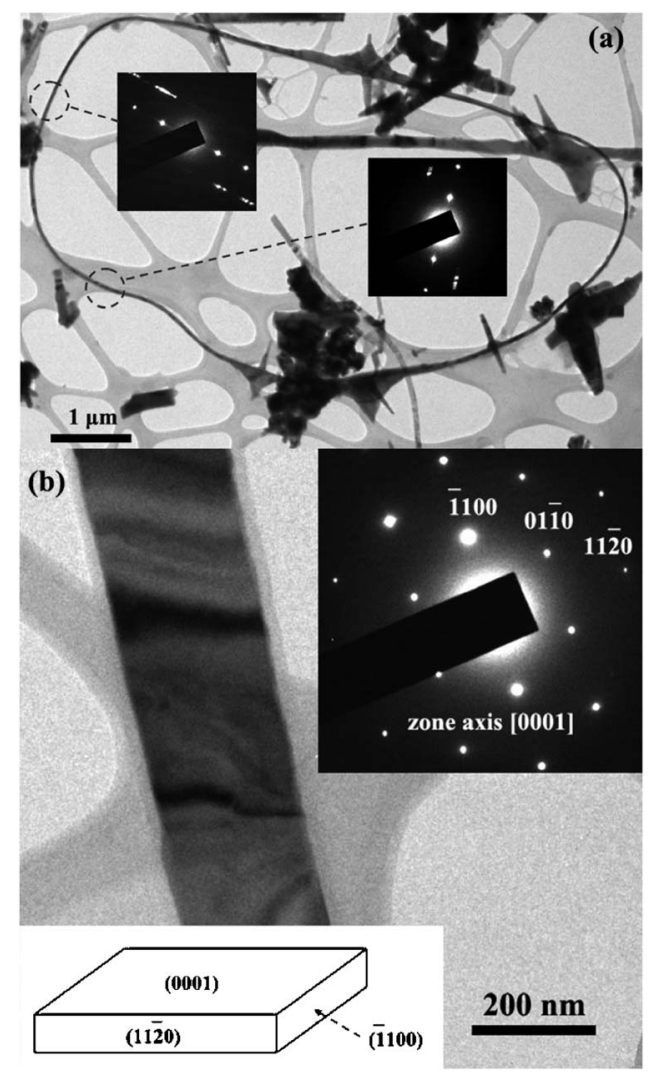

FIG. 3. (a) TEM image of a single ring. The insets are the SAED patterns of the corresponding regions of the ring as marked in the figure. (b) TEM image of a nanobelt forming the ring, the upper inset is the SAED taken at the center of the belt and the lower inset is the structure model of the $\mathrm{ZnSe}$ nanobelt.

grown at $1100{ }^{\circ} \mathrm{C},{ }^{23}$ while Jiang et al. reported the fabrication of wurtzite ZnSe nanoribbons. ${ }^{19} \mathrm{Li}$ suggested that the existence of hexagonal phase might probably be a result of high deposition temperature. Jiang briefly indicated that the formation of the hexagonal phase ribbons was started from the $\mathrm{ZnSe}$ nuclei which nucleated on bare Si substrate and continued to grow via the vapor-solid (VS) mechanism. In our work, we find that the growth pressure is a critical parameter for the formation of the $\mathrm{W}-\mathrm{ZnSe}$.

The formation of either the $\mathrm{ZB}$ or $\mathrm{W}$ structure depends on which type of nuclei dominates the substrate during nucleation. SAED measurements have been conducted in order to confirm the structure of the samples grown at 0.2 torr. In all the measured samples, only the $\mathrm{W}$-structured $\mathrm{ZnSe}$ is observed. We therefore conclude that the growth under lower reactor pressure favors the formation of wurtzite structured nuclei and hence the formation of the wurtzite structured rings as shown in Fig. 1. This phenomenon can be understood according to the Ostwald's step rule ${ }^{24}$ which describes that, under certain growth conditions, the metastable phase (i.e., the wurtzite phase in our case) would nucleate with a higher nucleation rate than that of the stable phase (i.e., the zinc-blende phase in our case). The Ostwald's step rule can be satisfied if the following condition holds: ${ }^{24}$

$$
\left(\frac{\Delta \mu_{s}}{\Delta \mu_{m}}\right)^{2}<\frac{b_{s}}{b_{m}}\left(\frac{\sigma_{s}}{\sigma_{m}}\right)^{3}\left(\frac{v_{s}}{v_{m}}\right)^{2},
$$

where $\Delta \mu$ is the supersaturation, $b$ is the geometric factor, $\sigma$ is the surface energy, and $v$ is the molecular volume. The subscripts $s$ and $m$ denote the stable and the metastable 


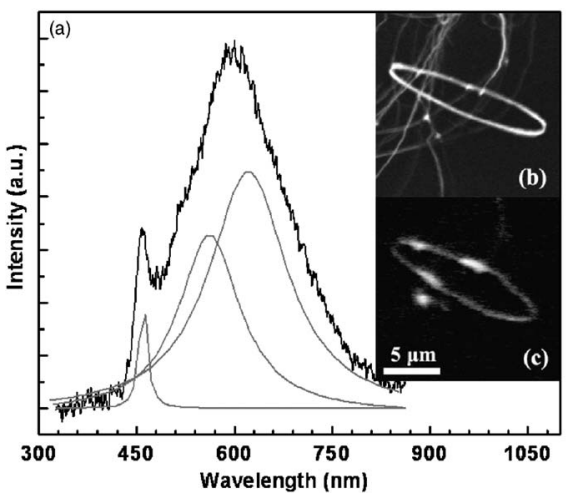

FIG. 4. (a) CL spectrum of the nanoring; (b) SEM image of a typical nanoring; (c) CL image of the nanoring.

phases respectively. Since $\Delta \mu_{s(m)}=(\mathrm{kT}) \ln \left[P / P_{s(m)}\right]$, the left hand side of Eq. (1) can be expressed as

$$
\frac{\Delta \mu_{s}}{\Delta \mu_{m}}=1+\ln \left(\frac{P_{m}}{P_{s}}\right) / \ln \left(\frac{P}{P_{m}}\right)
$$

where $T$ is the temperature, $k$ is the Boltzmann constant, and $P$ is the vapor pressure of the source material (i.e., $\mathrm{ZnSe}$ in our case). $P_{m}$ and $P_{s}$ are the equilibrium vapor pressure of the metastable and the stable phases, respectively. In our experiment, when the total reactor pressure increases, the evaporation rate of $\mathrm{ZnSe}$ powder reduces. Therefore, the vapor pressure of the $\mathrm{ZnSe}(P)$ decreases and, according to Eq. (2), $\left(\Delta \mu_{s} / \Delta \mu_{m}\right)$ increases. For a given phase, b, $\sigma$, and $v$ are constant. Therefore, as the reactor pressure decreases, the Ostwald's step rule is more probable to take place as Eq. (1). As a result, the metastable phase is more favorable to nucleate under a low reactor pressure which agrees with our experimental observations.

Once $\mathrm{W}-\mathrm{ZnSe}$ nuclei were formed, nanobelt would then grow via a VS mechanism. ${ }^{19}$ The growth of the ZnSe nanoring can be understood by the electrostatic polar charge model proposed by Kong and Wang. ${ }^{11}$ The wurtzite ZnSe crystal is described as alternating stacking of positively charged $\mathrm{Zn}$ and negatively charged Se ions along its $c$ axis. As a result, a dipole moment is set up due to the formation of positively charged $\mathrm{Zn}$-terminate and negatively charged Seterminate faces. In our case, the dipole moment is generated between the opposite sides of the nanobelts. In order to minimize the electrostatic energy of the entire system, the nanobelts will tend to coil up and neutralize the dipole moment by symmetric charge distribution. ${ }^{11}$

Figure 4 shows the CL spectrum, image, and the SEM image of a $\mathrm{ZnSe}$ nanoring. The $\mathrm{CL}$ peak at $\sim 2.7 \mathrm{eV}$ corresponds to the band edge transition of $\mathrm{ZnSe}$, while the emission with energy brand centered at $\sim 2.1 \mathrm{eV}$ can be referred to the self-activated (SA) emission. It has been reported that the low energy brand could correspond to the dopants of $\mathrm{Ga}$ and $\mathrm{P}^{25}$ However, it is not likely to be our case because our process has not utilized such dopants. The low energy brand can be resolved into two Lorentzian spectra as shown in Fig. 4(a) by gray lines. The peaks of the two Lorentzian spectra are $\sim 2$ and $2.2 \mathrm{eV} . \mathrm{N}$ and $\mathrm{N}_{2}$ doped $\mathrm{ZnSe}$ had the emission at $2.05 \mathrm{eV}{ }^{26}$ However, since an ion implantation method was used to dope $\mathrm{N}$ and $\mathrm{N}_{2}$ into $\mathrm{ZnSe}$ in reference. ${ }^{26}$ the emission could be due to the defects created by the implantation and may not be the effects of the dopants themselves.
An excess of $\mathrm{Zn}$ in $\mathrm{ZnSe}$ will result in the emission at $2.03 \mathrm{eV}$ (Ref. 27) which can be used to explain the emission centered at $2 \mathrm{eV}$ in our case. Concerning the emission at $2.2 \mathrm{eV}$, it can be explained by the dislocation, stacking faults, and nonstoichiometric defects reported in reference. ${ }^{28}$

In conclusion, wurtzite structured $\mathrm{ZnSe}$ nanorings have been fabricated. The formation of the metastable wurtzite phase is discussed and it is explained by the favorable nucleation of the metastable hexagonal phase under the Ostwald's step rule. The electrostatic polar charge model is used to explain the formation of the nanorings. The CL of the $\mathrm{ZnSe}$ nanorings with the band edge transition peak and broad emission at lower energy has been obtained.

One of the authors (W.C.H.C.) would like to acknowledge the support of UDF grant and seed funding of the University of Hong Kong. Another author (T.I.Y.) would like to acknowledge the support of small project funding. The authors would like to thank C. F. Mak for his technical assistance in the early stage of the project.

${ }^{1}$ J. Hu, T. W. Odom, and C. M. Lieber, Acc. Chem. Res. 32, 435 (1999).

${ }^{2}$ R. S. Wagner and W. C. Ellis, Appl. Phys. Lett. 4, 89 (1964).

${ }^{3}$ S. Y. Bae, H. W. Seo, J. Park, H Yang, and B. Kim, Chem. Phys. Lett. 376, 445 (2003)

${ }^{4}$ Y. P. Leung, Z. Liu, and S. K. Hark, J. Cryst. Growth 279, 248 (2005).

${ }^{5}$ Z. G. Yan, Y. W. Zhang, L. P. You, R. Si, and C. H. Yan, J. Cryst. Growth 262, 408 (2004).

${ }^{6}$ Z. H. Wu, X. Mei, D. Kim, M. Blumin, H. E. Ruda, J. Q. Liu, and K. L. Kavanagh, Appl. Phys. Lett. 83, 3368 (2003).

${ }^{7}$ T. Kuykendall, P. J. Pauzauskie, Y. Zhang, J. Goldberger, D. Sirbuly, J. Denlinger, and P. Yang, Nat. Mater. 3, 524 (2004).

${ }^{8}$ J. Q. Hu, Y. Bando, J. H. Zhan, M. Y. Liao, D. Golberg, X. L. Yuan, and T. Sekiguchi, Appl. Phys. Lett. 87, 113107 (2005).

${ }^{9}$ C. Wang, J Wang, Q. Li, and G. Yi, Adv. Funct. Mater. 15, 1471 (2005).

${ }^{10}$ Z. Q. Zhang, C. B. Jiang, S. X. Li, and S. X. Mao, J. Cryst. Growth 277, 321 (2005).

${ }^{11}$ X. Y. Kong and Z. L Wang, Nano Lett. 3, 1625 (2003).

${ }^{12}$ T. Matasuoka, Adv. Mater. (Weinheim, Ger.) 8, 469 (1996)

${ }^{13}$ R. Passler, E. Griebl, H. Ripel, G. Lautner, S. Bauer, H. Preis, W. Gebhardt, B. Buda, D. J. As, D. Schikora, K. Lischka, K. Papagelis, and S. Ves, J. Appl. Phys. 86, 4403 (1999).

${ }^{14}$ J. Wang, D. C. Hutchings, A. Miller, E. W. Vanstryland, K. R. Welford, I. T. Muirhead, and K. L. Lewis, J. Appl. Phys. 73, 4746 (1993).

${ }^{15}$ C. Natarajan, M. Sharon, C. L. Clement, and H. N. Spallart, Thin Solid Films 118, 234 (1994).

${ }^{16}$ P. V. Kamat and B. Patrick, J. Phys. Chem. 96, 6829 (1992).

${ }^{17}$ W. C. H. Choy, C. F. Guo, K. H. Pang, Y. P. Leung, G. Z. Wang, and K. W. Cheah, J. Nanosci. Nanotechnol. 6, 802 (2006).

${ }^{18}$ C. Ye, X. Fang, Y. Wang, P. Yan, J. Zhao, and L. Zhang, Appl. Phys. A: Mater. Sci. Process. 79, 113 (2004).

${ }^{19}$ Y. Jiang, X. M. Meng, W. C. Yiu, J. Liu, J. X. Ding, C. S. Lee, and S. T. Lee, J. Phys. Chem. B 108, 2784 (2004).

${ }^{20}$ W. C. H. Choy, C. F. Guo, Y. P Leung, G. Z. Wang, and T. I. Yuk, Appl. Phys. A: Mater. Sci. Process. 83, 301 (2006).

${ }^{21}$ H. Hartmann, R. Mach, and B. Selle, Current Topics in Materials Science, edited by E. Kaldis (North-Holland, New York, 1982), p. 9

${ }^{22}$ C. Y. Yeh, Z. W. Lu, S. Froyen, and A. Zunger, Phys. Rev. B 46, 10086 (1992).

${ }^{23}$ Q. Li., X. Gong, C. Wang, J. Wang, K. M. Ip, and S. K. Hark, Adv. Mater. (Weinheim, Ger.) 16, 1436 (2004).

${ }^{24}$ I. V. Markov, Crystal Growth for Beginners, 2nd Edition (World Scientific, Singapore 2003), Chap. 2.

${ }^{25}$ N. Sankar and K. Ramachandran, J. Cryst. Growth 247, 157 (2003).

${ }^{26}$ T. Yao, Z. Zhu, Y. H. Wu, C. D. Song, F. Nishiyama, K. Kimura, H. Kajiyama, S. Miwa, and T. Yasuda, J. Cryst. Growth 159, 214 (1996).

${ }^{27}$ V. V. Blinov, E. M. Gavrishchuk, V. G. Galstyan, V. S. Zimogorshii, I. A. Karetnikov, N. K. Morozova, and V. G. Plotnichenko, Inorg. Mater. 37, 1229 (2001).

${ }^{28}$ Z. T. Zhang, Z. Liu, Y. P. Leung, Q. Li, and S. K. Hark, Appl. Phys. Lett. 83, 5533 (2003) 